\title{
Time Optimal Path-Tracking Control of Kinematically Redundant Manipulators*
}

\author{
Shugen $\mathrm{MA}^{* *}$ and Mitsuru WATANABE***
}

\begin{abstract}
In this study, we propose a time optimal control scheme for kinematically redundant manipulators to track a predefined geometric path, subject to joint torque limits. The scheme can make full use of redundancy to increase the path-tracking velocity, and the time optimal trajectory planning problem is solved by using the phase-plane analysis and the linear programming technique. Computer simulation is also executed on a three-link planar rotary manipulator to show that, 1) the redundancy of the manipulator is fully used to increase the path-tracking velocity, and 2) redundant joints plus one more joint use their bound values of torque all the time while the time optimal path-tracking task is performed.
\end{abstract}

Key Words: Manipulator, Optimal Control, Motion Control, Redundancy, Time Optimal Path-Tracking Control, Maximum Path-Tracking Velocity, Torque Limit

\section{Introduction}

In this study, a time optimal control scheme is presented for kinematically redundant manipulators to track a predefined geometric path, subject to joint torque limits.

Kinematically redundant manipulators have been receiving an increasing attention from last decade because of their ability to perform tasks of high complexity. Besides the end-effector task, one or more additional tasks can be accomplished. Therein, the end-effector motion of the redundant manipulator was given as a primary task and the subtask such as the avoidance of singularities ${ }^{(1)}$, obstacles $^{(2),(3)}$, or joint limits ${ }^{(4)}$, the minimization of joint velocities $^{(5)}$ or joint torques ${ }^{(6),(7)}$, etc. was performed at same time. However, how to use the redundant degrees of freedom of the manipulator to increase the path-tracking velocity or to make the manipulator track the geometric path in minimum time, in subjection to joint torque limits, had never been discussed. It is physically more reasonable to find the possible path-tracking velocity of the manipulator, subject to joint torque limits. Many time optimal pathtracking control schemes of non-redundant manipulators were introduced for increasing the robot performance,

* Received 25th July, 2003 (No. 03-4100)

** Department of Systems Engineering, Faculty of Engineering, Ibaraki University, 4-12-1 Nakanarusawa-cho, Hitachi-shi, Ibaraki 316-8511, Japan. E-mail: shugen@dse.ibaraki.ac.jp

*** NEC Tokin Corporation, 6-7-1 Kohriyama, Taihaku-ku, Shendai-shi, Miyagi 316-8511, Japan. where the execution time was minimized, i.e., the maximal feasible path-tracking velocity was solved concerning with the manipulator limitations coming from the used actuators and the power-transmission mechanism ${ }^{(8)-(15)}$. However, only a few discussed the time optimal pathtracking control problem for the kinematically redundant manipulator $^{(16)}$, where an extended state space and a concept of the regular trajectory were used and the problem was solved by the Pontryagin's maximum principle ${ }^{(17)}$.

In this work, we discuss the time optimal trajectory planning problem for kinematically redundant manipulators. A time optimal path-tracking control scheme to make full use of redundancy to increase the path-tracking velocity, is proposed for kinematically redundant manipulators to track a predefined geometric path, subject to joint torque limits. The time optimal trajectory planning problem is solved by using the phase-plane analysis and the linear programming technique. Section 2 gives the basic problem formulation of the time optimal path-tracking control of manipulators. In section 3 , we firstly introduce the time optimal path-tracking control scheme for nonredundant manipulators, and then present the time optimal path-tracking control scheme of kinematically redundant manipulators. Computer simulation was also executed and its results are shown in section 4 . The conclusion of the paper is summarized at section 5 .

\section{Problem Formulation}

Consider a $n$-degrees-of-freedom manipulator with joint coordinates $\theta_{i}, i=1,2, \cdots, n$, and a task described by 
$m$ task coordinates $r_{j}, j=1,2, \cdots, m$ with $m \leq n$. With respect to $n$ joint coordinates $\boldsymbol{\theta}=\left[\theta_{1}, \theta_{2}, \cdots, \theta_{n}\right]^{T} \in \mathfrak{R}^{n}$ and its time-differentials (joint velocities and joint accelerations) $\dot{\boldsymbol{\theta}} \& \ddot{\boldsymbol{\theta}}$, the joint torques $\boldsymbol{\tau}=\left[\tau_{1}, \tau_{2}, \cdots, \tau_{n}\right]^{T} \in \mathfrak{R}^{n}$ of the manipulator can be given in a well-known closed form

$$
\boldsymbol{\tau}=\mathbf{M}_{(\boldsymbol{\theta})} \ddot{\boldsymbol{\theta}}+\boldsymbol{c}_{(\boldsymbol{\theta}, \dot{\boldsymbol{\theta}})}+\boldsymbol{g}_{(\boldsymbol{\theta})}
$$

where $\mathbf{M} \in \mathfrak{R}^{n \times n}$ is the inertia matrix, $\boldsymbol{c} \in \mathfrak{R}^{n}$ is the torque vector of Coriolis and centrifugal forces, and $\boldsymbol{g} \in \mathfrak{R}^{n}$ is the torque vector of gravity force, respectively. The joint torques, $\tau$, belong to the feasible set due to actuator efforts, $\Omega$, defined as

$$
\Omega=\left\{\tau \mid \tau_{i, \min } \leq \tau_{i} \leq \tau_{i, \max } ; i=1,2, \cdots, n\right\}
$$

On the other hand, the kinematic transformation of the manipulator from joint space to task space can be described by the following equations

$$
\boldsymbol{r}=\boldsymbol{p}(\boldsymbol{\theta}), \quad \dot{\boldsymbol{r}}=\mathbf{J}_{(\boldsymbol{\theta})} \dot{\boldsymbol{\theta}}, \quad \ddot{\boldsymbol{r}}=\mathbf{J}_{(\boldsymbol{\theta})} \ddot{\boldsymbol{\theta}}+\dot{\mathbf{J}}_{(\boldsymbol{\theta}, \dot{\boldsymbol{\theta}})} \dot{\boldsymbol{\theta}}
$$

where $\boldsymbol{r}=\left[r_{1}, r_{2}, \cdots, r_{m}\right]^{T} \in \mathfrak{R}^{m}, \dot{\boldsymbol{r}}$, and $\ddot{\boldsymbol{r}}$ are $m$-dimensional position vector, velocities, and accelerations in task space, $\boldsymbol{p} \in \mathfrak{R}^{m}$ is $m$-dimensional vector function representing direct kinematics, $\mathbf{J} \in \mathfrak{R}^{m \times n}$ is the Jacobian matrix, with elements given by $J_{i j}=\partial p_{i} / \partial \theta_{j}$, and $\mathbf{J} \in \mathfrak{R}^{m \times n}$ is its time derivative, respectively. Assume that the geometric path in task space is given in parameterized form as a vector function $\boldsymbol{f}(s) \in \mathfrak{R}^{m}$ of the scalar path parameter $s \in \mathfrak{R}$, $s_{0} \leq s \leq s_{T}$, where $\boldsymbol{f}\left(s_{0}\right)$ is the start point, and $\boldsymbol{f}\left(s_{T}\right)$ is the end point of the path. Thus,

$$
\boldsymbol{r}=\boldsymbol{f}(s), \quad \dot{\boldsymbol{r}}=\boldsymbol{f}_{(s)}^{\prime} \dot{s}, \quad \ddot{\boldsymbol{r}}=\boldsymbol{f}_{(s)}^{\prime} \ddot{s}+\boldsymbol{f}_{(s)}^{\prime \prime} \dot{s}^{2}
$$

Notation $x^{\prime}$ is used for differentiation with respect to $s$, $x^{\prime}=d x / d s$, and $\dot{x}$ is for differentiation with respect to time, $\dot{x}=d x / d t$, respectively.

To minimize motion time of the system (1) along a specified geometric path $\boldsymbol{f}(s), s_{0} \leq s \leq s_{T}$, we must solve the following problem:

$$
\min _{\tau \in \Omega} J=\int_{0}^{T} d t=\int_{s_{0}}^{s_{T}} \frac{d s}{\dot{s}}
$$

subject to system dynamics (1), path constraints (4), and joint torque constraints (2).

\section{Time Optimal Path-Tracking Control}

The motion of the manipulator is generally constrained by itself (due to joint torque limits coming from the used actuators). The problem is how to find a feasible trajectory from a given geometric path with simultaneous utilization of the maximal capabilities of the manipulator. The time optimal path-tracking problem of the nonredundant manipulator was widely discussed, and many time optimal path-tracking control methods were introduced. The time optimal path-tracking control problem of the redundant manipulator, however, was fairly discussed $^{(16)}$. In this section, we first introduce the time optimal path-tracking control scheme of non-redundant ma- nipulators and then present the time optimal path-tracking control method for kinematically redundant manipulators.

\subsection{In case of non-redundant manipulators}

For a non-redundant manipulator, $m=n$ and the rank of Jacobian matrix $\mathbf{J}$ equals $n$ except in the singular configurations. In the case, inverse of the Jacobian matrix exists and the parameterized path in joint coordinates can be derived by combining (3) and (4), given by

$$
\begin{aligned}
\boldsymbol{\theta}_{(s)}= & \boldsymbol{p}^{-1}(\boldsymbol{f}(s))=\hat{\boldsymbol{p}}^{-1}(s) \\
\dot{\boldsymbol{\theta}}_{(s, \dot{s})} & =\mathbf{J}_{(\boldsymbol{\theta}(s))}^{-1} \boldsymbol{f}_{(s)}^{\prime} \dot{s}=\hat{\mathbf{J}}_{(s)}^{-1} \boldsymbol{f}_{(s)}^{\prime} \dot{s} \\
\ddot{\boldsymbol{\theta}}_{(s, \dot{s}, \dot{s})} & =\mathbf{J}_{(\boldsymbol{\theta}(s))}^{-1}\left(\boldsymbol{f}_{(s)}^{\prime} \ddot{s}+\boldsymbol{f}_{(s)}^{\prime \prime} \dot{s}^{2}-\dot{\mathbf{J}}_{\left(\boldsymbol{\theta}(s), \dot{\boldsymbol{\theta}}_{(s, \dot{s}))}\right.} \dot{\boldsymbol{\theta}}_{(s, \dot{s})}\right) \\
& =\hat{\mathbf{J}}_{(s)}^{-1}\left(\boldsymbol{f}_{(s)}^{\prime} \ddot{s}+\boldsymbol{f}_{(s)}^{\prime \prime} \dot{s}^{2}-\dot{\hat{\mathbf{J}}}_{(s, \dot{s})} \dot{\boldsymbol{\theta}}_{(s, \dot{s})}\right)
\end{aligned}
$$

where $\boldsymbol{p}^{-1}$ means the inverse kinematics, and $\hat{\mathbf{J}}_{(s)} \& \dot{\hat{\mathbf{J}}}_{(s, \dot{s})}$

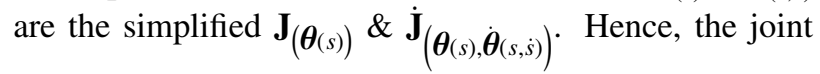
torques for tracking the path become to

$$
\tau_{(s, \dot{s}, \ddot{s})}=\zeta_{(s)} \ddot{s}+\boldsymbol{\eta}_{(s, \dot{s})}
$$

where,

$$
\begin{aligned}
& \boldsymbol{\zeta}_{(s)}=\mathbf{M}_{(\boldsymbol{\theta}(s))} \hat{\mathbf{J}}_{(s)}^{-1} \boldsymbol{f}_{(s)}^{\prime}=\hat{\mathbf{M}}_{(s)} \hat{\mathbf{J}}_{(s)}^{-1} \boldsymbol{f}_{(s)}^{\prime} \\
& \boldsymbol{\eta}_{(s, s)}=\hat{\mathbf{M}}_{(s)} \hat{\mathbf{J}}_{(s)}^{-1}\left(\boldsymbol{f}_{(s)}^{\prime \prime} \dot{s}^{2}-\dot{\hat{\mathbf{J}}}_{(s, s)} \dot{\boldsymbol{\theta}}_{(s, s)}\right)+\hat{\boldsymbol{c}}_{(s, s)}+\hat{\boldsymbol{g}}_{(s)}
\end{aligned}
$$

Therein, $\hat{\mathbf{M}}_{(s)}=\mathbf{M}_{(\boldsymbol{\theta}(s))}, \hat{\boldsymbol{c}}_{(s, \dot{s})}=\boldsymbol{c}_{\left(\boldsymbol{\theta}(s), \dot{\boldsymbol{\theta}}_{(s, s)}\right)}$ and $\hat{\boldsymbol{g}}_{(s)}=\boldsymbol{g}_{(\boldsymbol{\theta}(s))}$. Substituting (9) into (2) gives

$$
\tau_{i, \min } \leq \zeta_{i} \ddot{s}+\eta_{i} \leq \tau_{i, \max }
$$

The actuator-wise bounds on $\ddot{s}$ corresponding to $s$ and $\dot{s}$ are then derived as

$$
\begin{aligned}
& \ddot{s}_{i, \text { max }}= \begin{cases}\left(\tau_{i, \max }-\eta_{i}\right) / \zeta_{i} & \left(\zeta_{i}>0\right) \\
+\infty & \left(\zeta_{i}=0\right) \\
\left(\tau_{i, \min }-\eta_{i}\right) / \zeta_{i} & \left(\zeta_{i}<0\right)\end{cases} \\
& \ddot{s}_{i, \min }= \begin{cases}\left(\tau_{i, \min }-\eta_{i}\right) / \zeta_{i} & \left(\zeta_{i}>0\right) \\
-\infty & \left(\zeta_{i}=0\right) \\
\left(\tau_{i, \max }-\eta_{i}\right) / \zeta_{i} & \left(\zeta_{i}<0\right)\end{cases}
\end{aligned}
$$

The overall bounds are obtained by minimization and maximization over joints

$$
\ddot{s}_{\max }=\min _{i=1, \cdots, n} \ddot{s}_{i, \max }, \quad \ddot{s}_{\min }=\max _{i=1, \cdots, n} \ddot{s}_{i, \min }
$$

Note that these bounds depend on the state $[s, \dot{s}]$ and that for all admissible states the inequality $\ddot{s}_{\min } \leq \ddot{s}_{\max }$ must be fulfilled.

To find the optimal $s(t)$, let the scalar path acceleration be the control variable for the transformed problem: Find $\tilde{u}(t)$ which minimizes $T$ and drives the system 


$$
\ddot{s}=\tilde{u}(t)
$$

from $s_{0}$ with $\dot{s}_{0}=0$ to $s_{T}$ with $\dot{s}_{T}=0$ subject to $\ddot{s}_{\text {min }} \leq$ $\tilde{u}(t) \leq \ddot{s}_{\max }$. When the motion of the system is not at singular points and $\operatorname{arcs}(10),(18)$, the time optimal solution to the transformed problem is bang-bang in the control $\tilde{u}$. That is, at any instant, $\tilde{u}=\ddot{s}_{\min }$ or $\tilde{u}=\ddot{s}_{\max }$.

The time trajectory that describes the change of $\dot{s}$ with respect to $s$, can be generated by the phase-plane analysis technique ${ }^{(10)-(13),(15),(19),(20)}$. Solving the nonlinear equation $\ddot{s}_{\max }(s, \dot{s})=\ddot{s}_{\min }(s, \dot{s})$, the maximal admissible velocities $\dot{s}_{\max }$ with respect to $s$ were derived. Further on, through the recursive calculation from start point $\left(s_{0}, \dot{s}_{0}\right)$ to end point $\left(s_{T}, \dot{s}_{T}\right)$ and the recursive calculation backward from end point to start point, the path-tracking time trajectory was generated. The obtained time trajectory is a sequence of curves with maximal or minimal accelerations.

The above-stated time optimal control scheme would be not valid for kinematically redundant manipulators, since there exist the redundant degrees of freedom in the redundant manipulator, and the joint variables can not be only represented by the path-tracking parameter $s$ as (6), (7), and (8). Next we propose a new technique for solving the time optimal path-tracking problem of the redundant manipulator.

\subsection{In case of redundant manipulators}

For a kinematically redundant manipulator, $m<n$ and the inverse of Jacobian matrix does not exist. To resolve the kinematic redundancy of the redundant manipulator, the pseudoinverse-based formulation was widely used. However, for easily formulating the time optimal path-tracking control problem, the decomposed form of (3) is utilized in this study ${ }^{(21)}$. Rewriting (3) in the $\mathfrak{R}^{m}$ non-redundant set of joint variables, $\boldsymbol{\theta}_{n r}, \dot{\boldsymbol{\theta}}_{n r}, \ddot{\boldsymbol{\theta}}_{n r}$ in row space, and the $\mathfrak{R}^{n-m}$ redundant part of them, $\boldsymbol{\theta}_{r}, \dot{\boldsymbol{\theta}}_{r}, \ddot{\boldsymbol{\theta}}_{r}$ in null space, we have

$$
\begin{aligned}
\boldsymbol{r} & =\boldsymbol{p}(\boldsymbol{\theta})=\boldsymbol{p}\left(\boldsymbol{\theta}_{n r}, \boldsymbol{\theta}_{r}\right) \\
\dot{\boldsymbol{r}} & =\mathbf{J} \dot{\boldsymbol{\theta}}=\mathbf{J}_{n r} \dot{\boldsymbol{\theta}}_{n r}+\mathbf{J}_{r} \dot{\boldsymbol{\theta}}_{r} \\
\ddot{\boldsymbol{r}} & =\mathbf{J} \ddot{\boldsymbol{\theta}}+\dot{\mathbf{J}} \dot{\boldsymbol{\theta}} \\
& =\mathbf{J}_{n r} \ddot{\boldsymbol{\theta}}_{n r}+\mathbf{J}_{r} \ddot{\boldsymbol{\theta}}_{r}+\dot{\mathbf{J}}_{n r} \dot{\boldsymbol{\theta}}_{n r}+\dot{\mathbf{J}}_{r} \dot{\boldsymbol{\theta}}_{r}
\end{aligned}
$$

where $\mathbf{J}_{n r} \in \mathfrak{R}^{m \times m}$ is the full-rank determined Jacobian matrix corresponding to the non-redundant terms $\dot{\boldsymbol{\theta}}_{n r}$ in row space, and $\mathbf{J}_{r} \in \mathfrak{R}^{m \times(n-m)}$ is the over-determined Jacobian matrix corresponding to the redundant terms $\dot{\boldsymbol{\theta}}_{r}$ in null space, and $\dot{\mathbf{J}}_{n r}$ and $\dot{\mathbf{J}}_{r}$ are their time-derivatives, respectively. Substituting (4) for (13) and solving the nonredundant terms of joint variables, we have

$$
\begin{aligned}
\boldsymbol{\theta}_{n r} & =\boldsymbol{p}^{-1}\left(\boldsymbol{f}(s), \boldsymbol{\theta}_{r}\right)=\hat{\boldsymbol{p}}^{-1}\left(s, \boldsymbol{\theta}_{r}\right) \\
\dot{\boldsymbol{\theta}}_{n r} & =\mathbf{J}_{n r}^{-1}\left(\dot{\boldsymbol{r}}-\mathbf{J}_{r} \dot{\boldsymbol{\theta}}_{r}\right)=\hat{\mathbf{J}}_{n r}^{-1} \boldsymbol{f}^{\prime} \dot{s}-\hat{\mathbf{J}}_{n r}^{-1} \hat{\mathbf{J}}_{r} \dot{\boldsymbol{\theta}}_{r} \\
\ddot{\boldsymbol{\theta}}_{n r} & =\mathbf{J}_{n r}^{-1}\left(\ddot{\boldsymbol{r}}-\dot{\mathbf{J}}_{n r} \dot{\boldsymbol{\theta}}_{n r}-\mathbf{J}_{r} \ddot{\boldsymbol{\theta}}_{r}-\dot{\mathbf{J}}_{r} \dot{\boldsymbol{\theta}}_{r}\right) \\
& =\hat{\mathbf{J}}_{n r}^{-1} \boldsymbol{f}^{\prime} \ddot{s}+\hat{\mathbf{J}}_{n r}^{-1}\left(\boldsymbol{f}^{\prime \prime} \dot{s}^{2}-\dot{\mathbf{J}}_{n r} \hat{\mathbf{J}}_{n r}^{-1} \boldsymbol{f}^{\prime} \dot{s}\right)
\end{aligned}
$$

$$
-\hat{\mathbf{J}}_{n r}^{-1} \hat{\mathbf{J}}_{r} \ddot{\boldsymbol{\theta}}_{r}-\hat{\mathbf{J}}_{n r}^{-1}\left(\dot{\hat{\mathbf{J}}}_{r}-\dot{\hat{\mathbf{J}}}_{n r} \hat{\mathbf{J}}_{n r}^{-1} \hat{\mathbf{J}}_{r}\right) \dot{\boldsymbol{\theta}}_{r}
$$

where $\hat{\mathbf{J}}_{n r} \& \hat{\mathbf{J}}_{r}$ are function of the path-tracking parameter $s$ and the redundant terms $\boldsymbol{\theta}_{r}$, and are the simplified expression of $\mathbf{J}_{n r}\left(\boldsymbol{\theta}_{n r\left(s, \boldsymbol{\theta}_{r}\right)} \boldsymbol{\theta}_{r}\right) \& \mathbf{J}_{r\left(\boldsymbol{\theta}_{n r\left(s, \boldsymbol{\theta}_{r}\right)}, \boldsymbol{\theta}_{r}\right)}, \dot{\hat{\mathbf{J}}}_{n r} \& \dot{\hat{\mathbf{J}}}_{r}$ are function of the path-tracking parameter $s \& \dot{s}$ and the redundant terms $\boldsymbol{\theta}_{r} \& \dot{\boldsymbol{\theta}}_{r}$, and are the simplified expression of $\dot{\mathbf{J}}_{n r}\left(\boldsymbol{\theta}_{n r}\left(s, \boldsymbol{\theta}_{r}\right), \boldsymbol{\theta}_{r}, \dot{\boldsymbol{\theta}}_{n r}\left(s, \dot{s}, \boldsymbol{\theta}_{r}, \dot{\boldsymbol{\theta}}_{r}\right), \dot{\boldsymbol{\theta}}_{r}\right) \& \dot{\mathbf{J}}_{r\left(\boldsymbol{\theta}_{n r}\left(s, \boldsymbol{\theta}_{r}\right), \boldsymbol{\theta}_{r}, \dot{\boldsymbol{\theta}}_{n r}\left(s, \dot{s}, \boldsymbol{\theta}_{r}, \dot{\boldsymbol{\theta}}_{r}\right), \dot{\boldsymbol{\theta}}_{r}\right)}$, respectively. The non-redundant terms of joint variables are thus described by the path-tracking parameters $s, \dot{s}, \ddot{s}$ and the redundant terms $\boldsymbol{\theta}_{r}, \dot{\boldsymbol{\theta}}_{r}, \ddot{\boldsymbol{\theta}}_{r}$. By the same way, the system dynamics (1) can also be decomposed into two parts: one is corresponding to non-redundant set of joint variables, $\boldsymbol{\theta}_{n r}, \dot{\boldsymbol{\theta}}_{n r}, \ddot{\boldsymbol{\theta}}_{n r}$ in row space, and another is corresponding to redundant set of them, $\boldsymbol{\theta}_{r}, \dot{\boldsymbol{\theta}}_{r}, \ddot{\boldsymbol{\theta}}_{r}$ in null space. That is,

$$
\begin{aligned}
\boldsymbol{\tau} & =\mathbf{M}_{n r} \ddot{\boldsymbol{\theta}}_{n r}+\mathbf{M}_{r} \ddot{\boldsymbol{\theta}}_{r}+\boldsymbol{c}+\boldsymbol{g} \\
& =\hat{\mathbf{M}}_{n r} \ddot{\boldsymbol{\theta}}_{n r}+\hat{\mathbf{M}}_{r} \ddot{\boldsymbol{\theta}}_{r}+\hat{\boldsymbol{c}}+\hat{\boldsymbol{g}}
\end{aligned}
$$

where $\mathbf{M}_{n r} \in \mathfrak{R}^{n \times m}$ is the inertia matrix corresponding to the non-redundant accelerations $\ddot{\boldsymbol{\theta}}_{n r}$ in row space, $\mathbf{M}_{r} \in$ $\mathfrak{R}^{n \times(n-m)}$ is the inertia matrix corresponding to the redundant accelerations $\ddot{\boldsymbol{\theta}}_{r}$ in null space, $\hat{\mathbf{M}}_{n r}$ is the function of $s \& \boldsymbol{\theta}_{r}$ and the simplified expression of $\mathbf{M}_{n r}\left(\boldsymbol{\theta}_{n r\left(s, \boldsymbol{\theta}_{r}\right)}, \boldsymbol{\theta}_{r}\right), \hat{\mathbf{M}}_{r}$ is the function of $s \& \boldsymbol{\theta}_{r}$ and the simplified expression of $\mathbf{M}_{r\left(\boldsymbol{\theta}_{n r\left(s, \boldsymbol{\theta}_{r}\right)}, \boldsymbol{\theta}_{r}\right)}, \hat{\boldsymbol{c}}_{\left(s, \dot{s}, \boldsymbol{\theta}_{r}, \dot{\boldsymbol{\theta}}_{r}\right)}=\boldsymbol{c}_{\left(\boldsymbol{\theta}_{n r}\left(s, \boldsymbol{\theta}_{r}\right), \boldsymbol{\theta}_{r}, \dot{\boldsymbol{\theta}}_{n r}\left(s, \dot{s}, \boldsymbol{\theta}_{r}, \dot{\boldsymbol{\theta}}_{r}\right), \dot{\boldsymbol{\theta}}_{r}\right)}$, and $\hat{\boldsymbol{g}}_{\left(s, \boldsymbol{\theta}_{r}\right)}=\boldsymbol{g}_{\left(\boldsymbol{\theta}_{n r}\left(s, \boldsymbol{\theta}_{r}\right), \boldsymbol{\theta}_{r}\right)}$, respectively.

Substituting (14), (15), and (16) for (17), we have

$$
\boldsymbol{\tau}=\boldsymbol{\zeta}_{\left(s, \boldsymbol{\theta}_{r}\right)} \ddot{s}+\boldsymbol{\Gamma}_{\left(s, \boldsymbol{\theta}_{r}\right)} \ddot{\boldsymbol{\theta}}_{r}+\boldsymbol{\eta}_{\left(s, \dot{s}, \boldsymbol{\theta}_{r}, \dot{\boldsymbol{\theta}}_{r}\right)}
$$

where

$$
\begin{aligned}
\boldsymbol{\zeta}= & \hat{\mathbf{M}}_{n r} \hat{\mathbf{J}}_{n r}^{-1} \boldsymbol{f}^{\prime} \quad \in \mathfrak{R}^{n} \\
\boldsymbol{\Gamma}= & \left(\hat{\mathbf{M}}_{r}-\hat{\mathbf{M}}_{n r} \hat{\mathbf{J}}_{n r}^{-1} \hat{\mathbf{J}}_{r}\right) \quad \in \mathfrak{R}^{n \times(n-m)} \\
\boldsymbol{\eta}= & \hat{\mathbf{M}}_{n r} \hat{\mathbf{J}}_{n r}^{-1}\left[\boldsymbol{f}^{\prime \prime} \dot{\boldsymbol{S}}^{2}-\dot{\mathbf{J}}_{n r} \hat{\mathbf{J}}_{n r}^{-1} \boldsymbol{f}^{\prime} \dot{\boldsymbol{s}}\right. \\
& \left.+\left(\dot{\hat{\mathbf{J}}}_{n r} \hat{\mathbf{J}}_{n r}^{-1} \hat{\mathbf{J}}_{r}-\dot{\hat{\mathbf{J}}}_{r}\right) \dot{\boldsymbol{\theta}}_{r}\right]+\hat{\boldsymbol{c}}+\hat{\boldsymbol{g}} \quad \in \mathfrak{R}^{n}
\end{aligned}
$$

The joint torques $\tau$ are the function of the pathtracking parameters $s, \dot{s}, \ddot{s}$ and the redundant joint variables $\boldsymbol{\theta}_{r}, \dot{\boldsymbol{\theta}}_{r}, \ddot{\boldsymbol{\theta}}_{r}$. The time optimal path-tracking control problem of kinematically redundant manipulators that subject to joint torque limits, becomes to: $\min _{\tau \in \Omega} J=\int_{0}^{T} d t$, subject to $\boldsymbol{\tau}_{\min } \leq \boldsymbol{\zeta}_{\left(s, \boldsymbol{\theta}_{r}\right)} \ddot{\boldsymbol{s}}+\boldsymbol{\Gamma}_{\left(s, \boldsymbol{\theta}_{r}\right)} \ddot{\boldsymbol{\theta}}_{r}+\boldsymbol{\eta}_{\left(s, \dot{s}, \boldsymbol{\theta}_{r}, \dot{\boldsymbol{\theta}}_{r}\right)} \leq \boldsymbol{\tau}_{\max }$ and path constraints (4).

Same as the case of non-redundant manipulators, to find the optimal trajectory $s(t)$, let the scalar path acceleration be the control variable for the transformed problem: Find $\tilde{u}(t)$ which minimizes $T$ and drives the system

$$
\ddot{s}=\tilde{u}(t)
$$

from $s_{0}$ with $\dot{s}_{0}=0$ to $s_{T}$ with $\dot{s}_{T}=0$ subject to $\ddot{s}_{\text {min }} \leq$ $\tilde{u}(t) \leq \ddot{s}_{\max }$. When the motion of the system is not at singular points and $\operatorname{arcs}^{(10),(18)}$, the time optimal solution to 


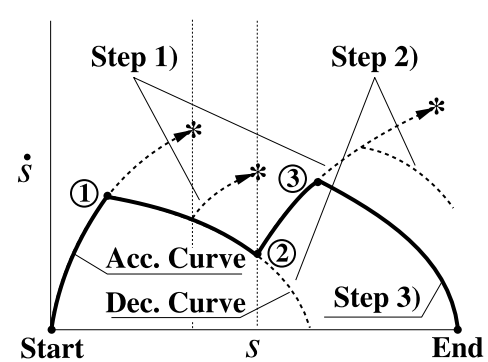

(a) Time trajectory $\dot{s}(s)$

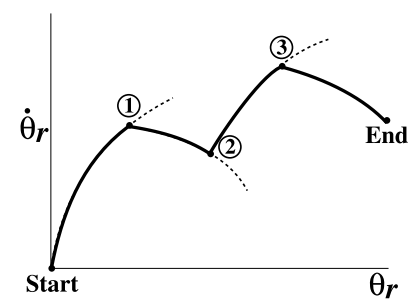

(b) Concept of the obtained $\dot{\boldsymbol{\theta}}_{r}\left(\boldsymbol{\theta}_{r}\right)$

Fig. 1 Generation of the path-tracking time trajectory and concept of the consequentlyobtained $\dot{\boldsymbol{\theta}}_{r}\left(\boldsymbol{\theta}_{r}\right)$ for the case of one redundant degree of freedom

the transformed problem must also be bang-bang in the control $\tilde{u}$. That is, at any instant, $\tilde{u}=\ddot{s}_{\min }$ or $\tilde{u}=\ddot{s}_{\max }$. Since all coefficients in (18) are known for any path position and velocity $(s, \dot{s})$ and redundant joint angles and velocities $\left(\boldsymbol{\theta}_{r}, \dot{\boldsymbol{\theta}}_{r}\right)$, the unknown $\ddot{\boldsymbol{s}}$ and $\ddot{\boldsymbol{\theta}}_{r}$, corresponding joint torques $\tau$ all appear linearly in (18). Hence, $\ddot{s}_{\text {min }}$ and $\ddot{s}_{\max }$ can be computed efficiently as the solution to a standard linear programming problem.

\subsubsection{Feasible path-tracking acceleration of re-} dundant manipulators The problem to obtain the feasible path-tracking acceleration of kinematically redundant manipulators that subject to joint torque limits can be seen as a linear programming problem. The feasible path-tracking acceleration is thus derived by the linear programming technique. That is, solving the linear programming problem

Basic variables: $\ddot{\boldsymbol{\theta}}_{r}, \ddot{s}\left(\ddot{\theta}_{m+1}, \cdots, \ddot{\theta}_{n}, \ddot{s}\right)$

Objective function: $J=\ddot{s} \quad$ (Minimization or Maximization)

Constraints: $\boldsymbol{\tau}_{\min } \leq \boldsymbol{\zeta} \ddot{\boldsymbol{s}}+\boldsymbol{\Gamma} \ddot{\boldsymbol{\theta}}_{r}+\boldsymbol{\eta} \leq \boldsymbol{\tau}_{\max }$

to obtain the feasible path-tracking acceleration $\ddot{s}$. Beside the maximum value $\ddot{s}_{\max }$ (obtained through maximization of the objective function $J$ ) of the feasible acceleration and its minimum value $\ddot{s}_{\min }$ (obtained through minimization of the objective function $J$ ), the accelerations of redundant joints $\ddot{\boldsymbol{\theta}}_{r}$ are also derived at same time. Substituting these values for (16), we can have the accelerations of non-redundant joints $\ddot{\boldsymbol{\theta}}_{n r}$. Thus, the joint accelerations $\ddot{\boldsymbol{\theta}}$ that generate the feasible path-tracking accelerations are obtained. It should be noted again that, $\zeta, \Gamma$, and $\boldsymbol{\eta}$ are the function of $s, \dot{\boldsymbol{s}}$, and $\boldsymbol{\theta}_{r}, \dot{\boldsymbol{\theta}}_{r}$. Only after the values of $s, \dot{s}$, and $\boldsymbol{\theta}_{r}, \dot{\boldsymbol{\theta}}_{r}$ are known, the problem becomes to a linear programming problem and can be solved by the linear programming technique. The joint velocities $\dot{\boldsymbol{\theta}}$ and joint coordinates $\boldsymbol{\theta}$, same as $s$ and $\dot{\boldsymbol{s}}$, are derived through integration, starting from their initial values of $\boldsymbol{\theta}_{\mid t=0}=\boldsymbol{\theta}_{0}$ and $\dot{\boldsymbol{\theta}}_{\mid t=0}=\dot{\boldsymbol{\theta}}_{0}$.

\subsubsection{Generation of path-tracking time trajectory} Same as the case of non-redundant manipulators, the pathtracking time trajectory from the start point $\left(s_{0}, \dot{s}_{0}\right)$ (generally $\left.\dot{s}_{0}=0\right)$ to the end point $\left(s_{T}, \dot{s}_{T}\right)\left(\right.$ generally $\left.\dot{s}_{T}=0\right)$ is generated by the $s-\dot{s}$ phase-plane analysis. The maximal admissible velocities $\dot{s}_{\max }$ with respect to $s$, however, are difficultly derived and the backward calculation (from end to start) is not valid, since the redundant manipulator has redundant degrees of freedom or extra variables $\ddot{\boldsymbol{\theta}}_{r}$ and the joint velocities $\dot{\boldsymbol{\theta}}$ and its coordinates $\boldsymbol{\theta}$ are possibly derived only by integration. Only calculation in regular order (from start to end) is thus possibly used. In this paper, we propose a time-trajectory generation algorithm where only regular-order calculation is utilized.

In $s-\dot{s}$ phase plane, at first calculate the path-tracking velocity accelerated in feasible maximum acceleration from start point $\left(s_{0}, \dot{s}_{0}\right)$ with the initial values of joint variables $\left(\boldsymbol{\theta}_{0}, \dot{\boldsymbol{\theta}}_{0}\right)$ until $\ddot{s}_{\text {max }}=\ddot{s}_{\text {min }}$, to obtain the 'acceleration curve'. Then, from the point on the acceleration curve with the corresponding $\boldsymbol{\theta}$ and $\dot{\boldsymbol{\theta}}$ calculate the 'deceleration curve' in feasible minimum acceleration, to find out the deceleration curve that crosses with $s$-axis in maximum value of $s$-coordinate and makes inequality $\ddot{s}_{\max }<\ddot{s}_{\min }$ never happen. If the obtained deceleration curve passes over the end point $\left(s_{T}, \dot{s}_{T}\right)$, then find out the deceleration curve that passes through the end point and finish the calculation; If not, perform the following procedures. Here and in follows, we call the intersection '*' of the acceleration curve with $\ddot{s}_{\text {max }}=\ddot{s}_{\text {min }}$ boundary curve as "Inadmissible point".

Step 1) From the point with the corresponding $\boldsymbol{\theta}$ and $\dot{\boldsymbol{\theta}}$, on the deceleration curve with respect to $s$-coordinate of the inadmissible point ' $*$ ', calculate the path-tracking velocity accelerated in feasible maximum acceleration until $\ddot{s}_{\text {max }}=\ddot{s}_{\text {min }}$, to obtain new inadmissible point. If $\dot{s}$ of the newly-obtained inadmissible point is larger than that of the previously-obtained inadmissible point, or if $s$-coordinate of the newly-obtained inadmissible point is larger than that of the crossing point of the deceleration curve with $s$-axis, perform Step 2). Otherwise, perform this Step 1) recursively.

Note that, starting the calculation again from the point on the deceleration curve that is with respect to $s-$ coordinate of the inadmissible point '*' is for reducing the calculation steps and speeding up the calculation. 
Step 2) Go back on the acceleration curve and from the point on the acceleration curve with the corresponding $\boldsymbol{\theta}$ and $\dot{\theta}$ calculate the deceleration curve that crosses with $s$-axis in maximum value of $s$-coordinate while $\ddot{s}_{\max } \geq$ $\ddot{s}_{\text {min }}$ always hold. If the deceleration curve is not passing over the end point $\left(s_{T}, \dot{s}_{T}\right)$, then perform Step 1); otherwise perform Step 3).

Step 3) Find out the deceleration curve that passes through the end point $\left(s_{T}, \dot{s}_{T}\right)$ and connect sequence of the obtained acceleration and deceleration curves to have the time trajectory. At the same time as we have the time trajectory $\dot{s}(s)$, the corresponding manipulator posture $\boldsymbol{\theta}$ and the joint velocities $\dot{\theta}$ are also obtained.

It should be noted here that, the above algorithm generates a more efficient time trajectory, but not an exact minimum-time one. Since the exact minimum time trajectory has many switching and the joint torque inputs are not smooth ${ }^{(16)}$, it is not feasible for the control of real manipulators. The switching has been consequently reduced as little as possible in the proposed algorithm by 1) starting the calculation from the point on the deceleration curve corresponding to $s$-coordinate of the inadmissible point '*, and 2) calculating the deceleration curve that crosses with $s$-axis in maximum value of $s$-coordinate while $\ddot{s}_{\max } \geq \ddot{s}_{\min }$ always hold. This point is an advantage of the proposed algorithm in comparison with the previously-proposed algorithm ${ }^{(16)}$. In addition, we have not considered the final joint velocities in the algorithm. The redundancy of the manipulator is used to increase locally the path-tracking velocity, but the final joint velocities can not be guaranteed zero. A proper initial manipulator posture can be selected by search to solve the problem, it however needs enormous calculation time. The algorithm proposed in this study only gives the results without adjusting the initial manipulator posture. How to effectively adjust the initial manipulator posture will be discussed in coming studies.

\section{Computer Simulation}

To demonstrate the effectiveness of the proposed time optimal path-tracking control scheme of kinematically redundant manipulators, computer simulation was performed on a simple planner redundant manipulator as shown in Fig. 2, where the gravity was neglected. The manipulator model has three rotary joints where the orientation of the end-effector is not constrained and one degree of redundancy is given. The links labeled 1, 2, 3 from the base of the manipulator, are all identical and modeled as uniform thin rods with length $\left(\ell_{i}=1.0[\mathrm{~m}]\right)$ and mass $\left(m_{i}=10.0[\mathrm{~kg}]\right)$. The joint torque limits due to actuator efforts are set as $\pm 10[\mathrm{Nm}]$. As the desired geometric path of the end-effector, we utilized the straightline Cartesian trajectories given in the parametric form, defined by $\boldsymbol{f}(s)=\left[x_{0}+\cos (\alpha) s, y_{0}+\sin (\alpha) s\right]^{T},\left(\dot{s}_{0}=\dot{s}_{E}=\right.$
Table 1 The resulted motion time for eight paths

\begin{tabular}{|c||c|c|c|c|}
\hline \hline Angle $\alpha[\mathrm{rad}]$ & 0 & $\pi / 4$ & $\pi / 2$ & $3 \pi / 4$ \\
\hline Time $T[\mathrm{sec}]$ & 1.79 & 2.00 & 2.17 & 2.35 \\
\hline \hline Angle $\alpha[\mathrm{rad}]$ & $\pi$ & $5 \pi / 4$ & $3 \pi / 2$ & $7 \pi / 4$ \\
\hline Time $T[\mathrm{sec}]$ & 1.91 & 1.84 & 1.55 & 1.49 \\
\hline \hline
\end{tabular}

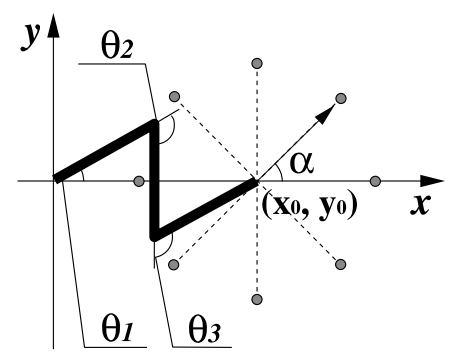

Fig. 2 Manipulator model used in the computer simulation

$0)$. Therein, $\left(x_{0}, y_{0}\right)$ is the initial manipulator end position derived from initial manipulator arm posture $\theta_{1}=$ $\left.\pi / 6[\mathrm{rad}], \theta_{2}=-2 \pi / 3[\mathrm{rad}], \theta_{3}=2 \pi / 3[\mathrm{rad}]\right)$ with $\dot{\boldsymbol{\theta}}_{(0)}=\mathbf{0}$. Eight paths were selected for the manipulator to track along, the length of each path is $1 \mathrm{~m}$ and their inclination angles $\alpha$ are $0, \pi / 4, \pi / 2,3 \pi / 4, \pi, 5 \pi / 4,3 \pi / 2,7 \pi / 4$ [rad], respectively. The resulted motion time was listed in Table 1. Figure 3 shows the corresponding joint velocities for the cases of $\alpha=3 \pi / 4$ and $\alpha=5 \pi / 4$ as an example. From the results, it is known that, the longer motion time has been taken for the case of $\alpha=3 \pi / 4$ with the fast-increased joint velocities, in comparison with the case of $\alpha=5 \pi / 4$. As a result, we know that the longer motion time is necessary for the case that the manipulator arm moves into the postures where the joint velocities are fast increased. The joint torques due to the joint velocities thus become so high that only a small room of joint torques is left for increasing the path-tracking velocities. The proposed time optimal path-tracking scheme does not behaves the function of preventing the increase of joint velocities.

One example of the derived time-trajectory, the consequently-generated change of $\dot{\theta}_{r}\left(\dot{\theta}_{3}\right)$ with respect to $\theta_{r}\left(\theta_{3}\right)$, and the generated arm motion, where $\alpha=5 \pi / 4$, are shown in Fig. 4, and the corresponding torque profiles of joints 1, 2, 3 are shown in Fig. 5, respectively. As seen, two joint used their bound value all the time while the manipulator tracking the path in the maximal velocity, and only one switching exists in the generated time trajectory. From section 3.2.1, it is known that there have $n-m+1$ basic variables in the linear programming problem. The largest number of saturated actuators should be $n-m+1$ (redundant ones $(n-m)$ plus one more $\left.{ }^{(16)}\right)$. For the example that we used here, $n-m+1=3-2+1=2$ ( 1 redundancy and 1). As a result, we know that the redundancy of the manipulator is effectively utilized to increase the pathtracking velocity, with feasible joint torque inputs. However, the joint velocities at end of motion are not zero, as 
Joint 1

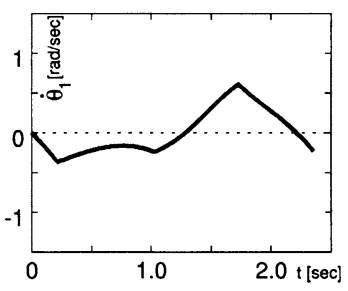

Joint 2

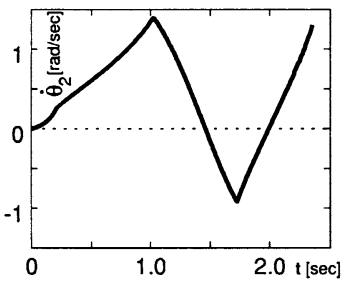

Joint 3

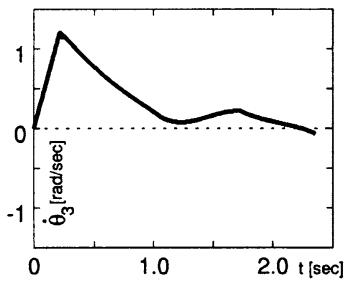

(a) Joint velocities for the case of $\alpha=3 \pi / 4$

Joint 1

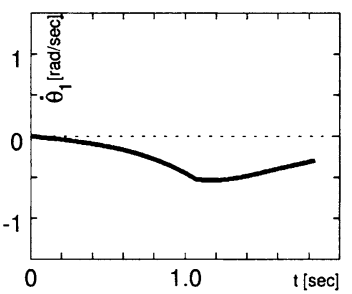

Joint 2

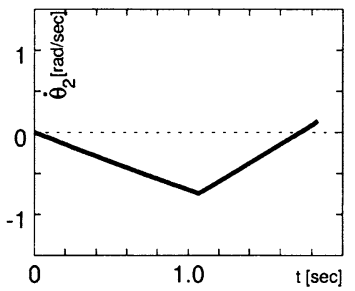

Joint 3

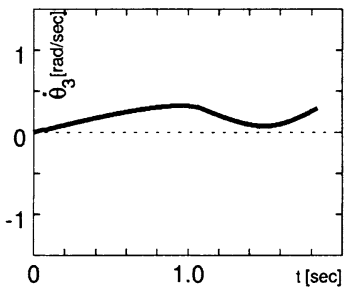

(b) Joint velocities for the case of $\alpha=5 \pi / 4$

Fig. 3 Joint velocities for the cases where $\alpha=3 \pi / 4$ and $\alpha=5 \pi / 4$

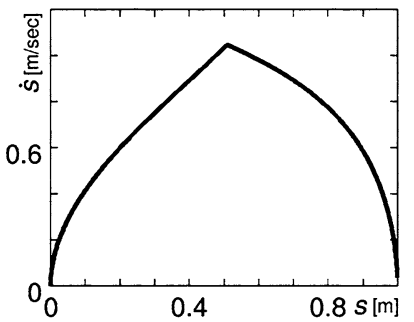

(a) Time trajectory

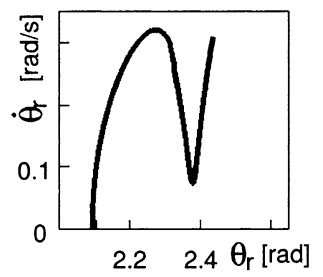

(b) Profile of $\dot{\theta}_{r}$ w.r.t. $\theta_{r}$

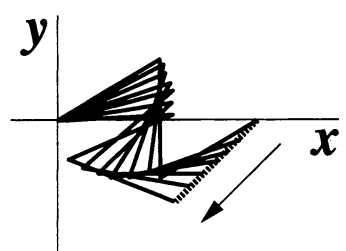

(c) Arm motion

Fig. 4 The generated time trajectory, the change of $\dot{\theta}_{r}\left(\dot{\theta}_{3}\right)$ with respect to $\theta_{r}\left(\theta_{3}\right)$, and the corresponding arm motion in case that $\alpha=5 \pi / 4$

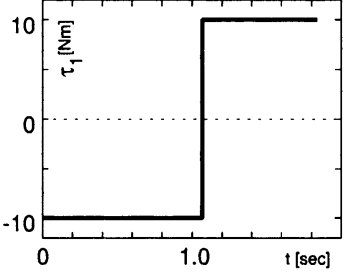

(a) Joint 1

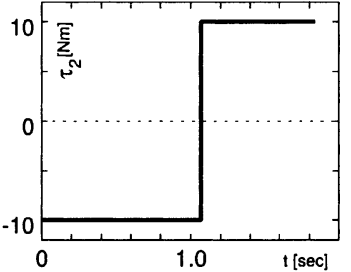

(b) Joint 2

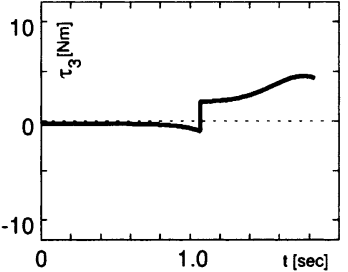

(c) Joint 3

Fig. 5 Profile of joint torques corresponding to the arm motion shown in Fig. 4

shown in Figs. 3 and 4(b). This is a critical point same as that in the local dynamic redundancy resolution control of redundant manipulators where the dynamics of the manipulator was taken into account. Since the derived trajectory from start to end and the derived trajectory from end to start are not continuously connected as shown in Fig. 6, the backward calculation (from end to start) is not valid. If this discontinued time trajectory was used, the infinitely-large pulse torques at the instant must be needed for the manipulator to track the path. It is not feasible for the control of real manipulators. Therefore, one considerable technique to guarantee the joint velocities at end of motion be zero would be by selecting a proper initial manipulator posture. Figures 7 and 8 shows one example where the initial manipulator posture was adjusted to make the joint velocities at end of motion zero. To select the initial manipulator posture to make the joint velocities at end of motion zero, however, needs enormous calculation time. In addition, from Figs. 5 and 8, we also know that the resulted motion time $(2.53 \mathrm{sec})$ with adjusting the initial manipulator posture is larger than that $(1.84 \mathrm{sec})$ without adjusting the initial manipulator posture. In order to make full use of redundancy to increase the path-tracking velocity, we only discuss in this study the results without 

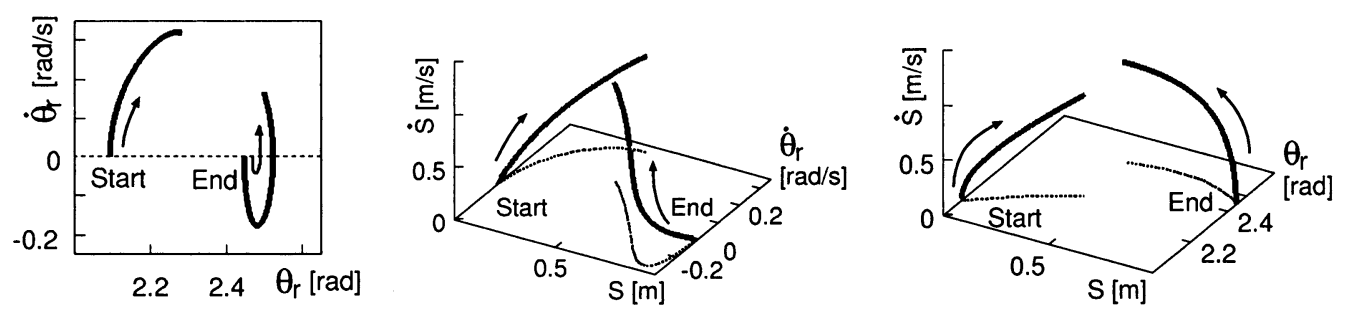

Fig. 6 Profile of $\dot{\theta}_{r}$ with respect to $\theta_{r}$, profile of $\dot{s}$ with respect to $s$ and $\theta_{r}$, profile of $\dot{s}$ with respect to $s$ and $\dot{\theta}_{r}$

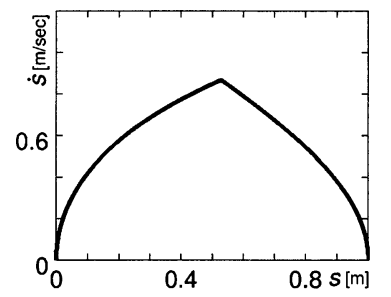

(a) Time trajectory

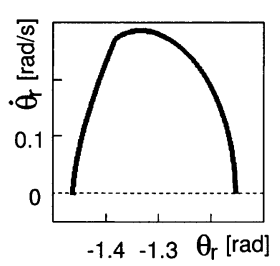

(b) Profile of $\dot{\theta}_{r}$ w.r.t. $\theta_{r}$

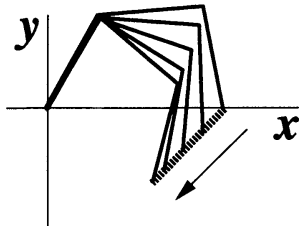

(c) Arm motion

Fig. 7 The generated time trajectory, the change of $\dot{\theta}_{r}\left(\dot{\theta}_{3}\right)$ with respect to $\theta_{r}\left(\theta_{3}\right)$, and the corresponding arm motion while the initial manipulator posture was adjusted, in case that $\alpha=5 \pi / 4$

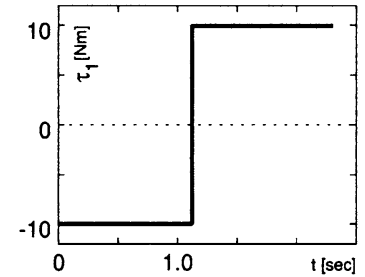

(a) Joint 1

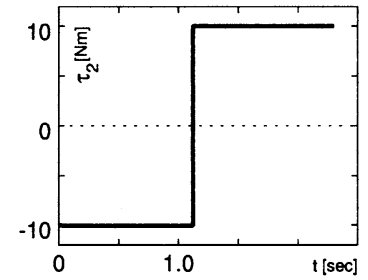

(b) Joint 2

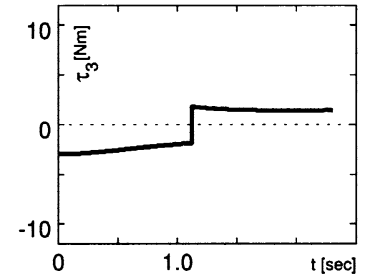

(c) Joint 3

Fig. 8 Profile of joint torques corresponding to the arm motion shown in Fig. 7

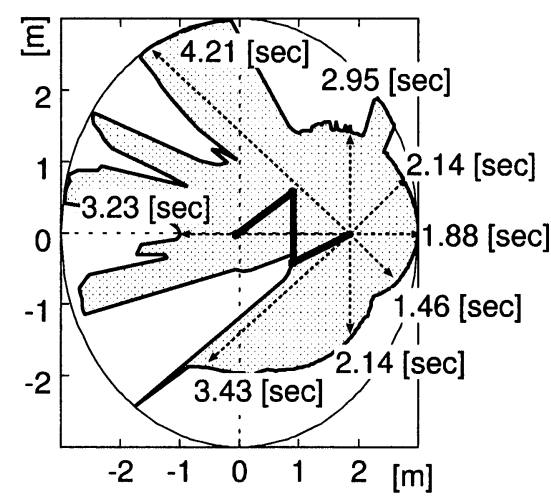

(a) Area map

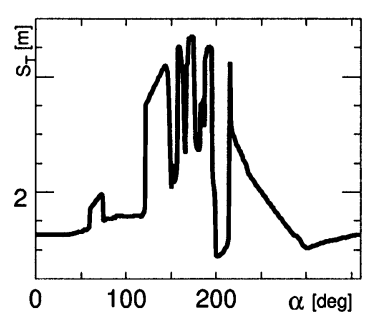

(b) Limit distances w.r.t $\alpha$

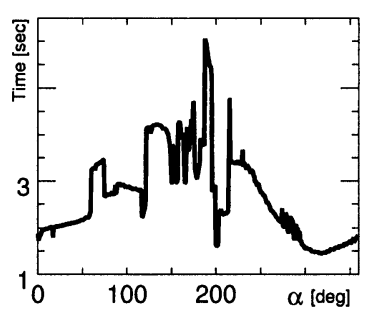

(c) Motion time w.r.t $\alpha$

Fig. 9 Boundary of the straight-line paths and the corresponding path-tracking motion time

adjusting the initial manipulator posture as most of the dynamic redundancy resolution control scheme, even though the zero end-motion velocities of joints are very important in real use of manipulators. The joints at end of motion, however, could be stopped by a break in case of only one path-tracking task, or the joint velocities at end of motion could be seen as the initial condition of next path-tracking task in case of continuously performing next path-tracking task.

Figure 9 shows the boundary of the path that the manipulator can track by the proposed time optimal path-tracking control scheme, and the corresponding path-tracking motion times for the inclination angles $\alpha$ : $0, \pi / 4, \pi / 2,3 \pi / 4, \pi, 5 \pi / 4,3 \pi / 2,7 \pi / 4[\mathrm{rad}]$. For clarity, the change of the limit distance from initial manipulator end position and the corresponding path-tracking motion time 
Joint 1

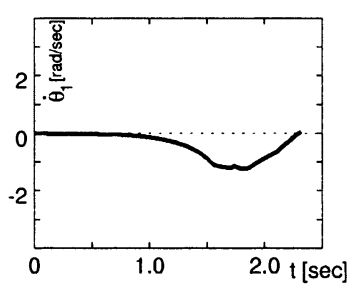

Joint 2

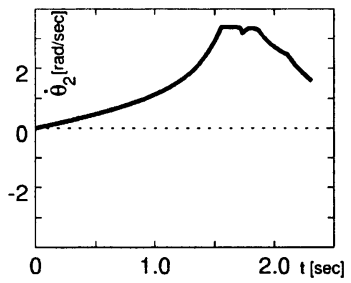

Joint 3

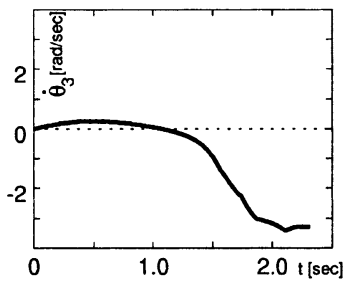

$\pi / 2$
Joint 1

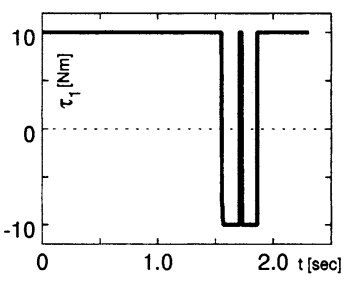

Joint 2

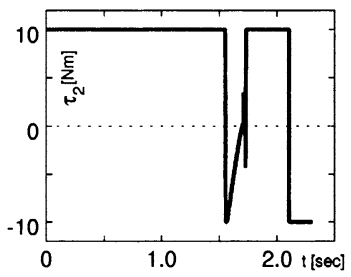

Joint 3

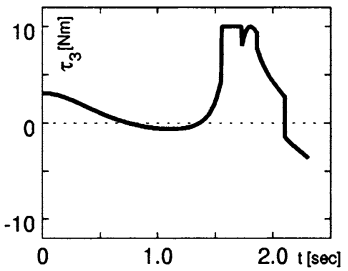

(b) Joint torques for the case of $\alpha=\pi / 2$

Fig. 10 Joint velocities and corresponding joint torques for the cases where $\alpha=\pi / 2$

with respect to the inclination angle $\alpha$ are also shown in Fig. 9. From the results, we know that, the proposed time optimal path-tracking control scheme has limitation, in which the path for the manipulator to track in minimum time can not cover all possible motion area if we do not adjust the initial manipulator posture, it is directiondependent. The reason would be that, the proposed minimum time path-tracking scheme can not prevent the increase of joint velocities, as shown in Fig. 10. The high joint velocities make the joint torques all meet their limit value as shown in Fig. 10 (at $t=1.54$ [sec]) and the pathtracking velocities never be further accelerated. Therefore, while the geometric path is far away from the arm base, the time trajectory can not be generated by the proposed scheme and some paths can not be tracked by the redundant manipulator. It should be pointed out that, if we adjust the initial manipulator posture, the manipulator can track all the paths in the possible motion area.

At last, we give the result obtained by comparison of the proposed time optimal control scheme with the pseudoinverse-based torque optimization technique ${ }^{(6)}$. In the computer simulation, same arm parameters were used, and the path parameters (same straight Cartesian paths whose lengths are $1 \mathrm{~m}$ and their inclination angles $\alpha$ are $0, \pi / 4, \pi / 2,3 \pi / 4, \pi, 5 \pi / 4,3 \pi / 2,7 \pi / 4$ [rad], starting and ending with zero velocity, constant bang-bang type acceleration) were chosen. The motion time derived by the proposed time optimal control scheme has been utilized. From the obtained joint torques for each motion, as shown in Table 2, it is known that at least one joint torque is larger than $10 \mathrm{Nm}$. The joint torques obtained by the proposed scheme are always under $10 \mathrm{Nm}$, as shown in Fig. 5. In other words, to track well the same path by local torque optimization scheme while holding the joint torques under
Table 2 Maximum value of joint torques for eight paths

\begin{tabular}{|r||c|c|c|c|}
\hline \hline Angle $\alpha[\mathrm{rad}]$ & 0 & $\pi / 4$ & $\pi / 2$ & $3 \pi / 4$ \\
\hline Max. Torque $\tau_{\max }[\mathrm{Nm}]$ & 14.99 & 323.95 & 88.85 & 1757.88 \\
\hline \hline Angle $\alpha[\mathrm{rad}]$ & $\pi$ & $5 \pi / 4$ & $3 \pi / 2$ & $7 \pi / 4$ \\
\hline Max. Torque $\tau_{\max }[\mathrm{Nm}]$ & $\infty$ & 27.46 & 28.81 & 27.74 \\
\hline \hline
\end{tabular}

their limits needs more longer time than that by the proposed scheme. Thus, we know that our time optimal control scheme is better in effective use of joint torques than the traditional local torque optimization control schemes.

\section{Conclusion}

In this study, we presented a time optimal control scheme for kinematically redundant manipulators to track a predefined geometric path, subject to joint torque limits. Since the redundant manipulator has redundant degrees of freedom, the technique for non-redundant manipulator would be not yet valid. The maximal admissible velocities are difficultly derived and the backward calculation (from end to start) is not possible. Only calculation in regular order (from start to end) is possibly used in the case of kinematically redundant manipulators. A time-generation algorithm where only regular-order calculation (from start to end) is used, was proposed for kinematically redundant manipulators, and the time optimal trajectory planning problem was solved by using the phase-plane analysis and the linear programming technique. Computer simulation was also executed on a three-link planar rotary manipulator to show that, 1) the proposed scheme makes the redundancy of the manipulator fully used to increase the path-tracking velocity, and 2) two joints (redundant joint plus one more joint) use their bound values of torque all the time while the time optimal path-tracking task is per- 
formed. From computer simulation, we have also known that, the path tracked by the manipulator in the proposed time optimal path-tracking control scheme can not cover all possible motion area. However, through adjusting the initial manipulator posture, this problem can be solved and the manipulator can track all the paths in the possible motion area.

\section{References}

( 1 ) Nakamura, Y. and Hanafusa, H., Inverse Kinematics Solutions with Singularity Robustness for Robot Manipulator Control, Trans. of ASME J. of Dynamics Systems, Measurement, and Control, Vol.108 (1986), pp.163-171.

( 2 ) Maciejewski, A.A. and Klein, C.A., Obstacle Avoidance for Kinematically Redundant Manipulators in Dynamically Varying Environments, Int. J. of Robotics Research, Vol.4, No.3 (1985), pp.109-117.

( 3 ) Nakamura, Y., Hanafusa, H. and Yoshikawa, T., Task Priority Based on Redundancy Control of Robot Manipulator, Int. J. of Robotics Research, Vol.6, No.2 (1987), pp.3-15.

(4) Liegeois, A., Automatic Supervisory Control of the Configuration and Behavior of Multi-Body Mechanisms, IEEE Trans. on Sys., Man, and Cybernetics, Vol.7, No.12 (1977), pp.868-871.

( 5 ) Whitney, D.E., Resolved Motion Control of Manipulator and Human Prosthesis, IEEE Trans. on ManMachine Systems, Vol.10, No.2 (1969), pp.47-53.

( 6 ) Kazerounian, K. and Nedungadi, A., Redundancy Resolution of Robotic Manipulators at the Acceleration Level, Proc. 7th IFToMM World Congress, Sevilla, Spain, (1987), pp.1207-1211.

( 7 ) Ma, S., A Balancing Technique to Stabilize Local Torque Optimization Solution of Redundant Manipulators, Journal of Robotic Systems, Vol.13, No.3 (1996), pp.177-185.

( 8 ) Hollerbach, J.M., Dynamic Scaling of Manipulator Trajectories, Trans. of ASME, J. of Dyn. Sys. Meas. and Contr., Vol.106 (1984), pp.102-106.

(9) Shiller, Z. and Dubowsky, S., On the Optimal Control of Robotic Manipulators with Actuator and EndEffector Constraints, Proc. IEEE Int. Conf. on Robotics and Automation, Nagoya, Japan, (1985), pp.614-620.

(10) Shiller, Z. and Lu, H., Computation of Path Con- strained Time Optimal Motions with Dynamic Singularities, Trans. of ASME, J. of Dyn. Sys. Meas. and Contr., Vol.114 (1992), pp.34-40.

(11) Bobrow, J.E., Dubowsky, S. and Gibson, J.S., TimeOptimal Control of Robotic Manipulators along Specified Paths, Int. J. of Robotic Research, Vol.4, No.3 (1985), pp.3-17.

(12) Shin, K.G. and McKay, N.D., Minimum-Time Control of Robotic Manipulators with Geometric Path Constraints, IEEE Trans. on Auto. Contr., Vol.AC-30, No.6 (1985), p.531.

(13) Pfeifer, F. and Johanni, R., A Concept for Manipulator Trajectory Planning, Proc. IEEE Int. Conf. on Robotics and Automation, San Francisco, California, (1986), pp.1399-1405.

(14) Huang, H.P. and McClamroch, N.H., Time-Optimal Control for a Robotic Contour Following Problem, IEEE Trans. on Robotics and Automation, Vol.4, No.2 (1988), pp.140-148.

(15) Zlajpah, L., On Time Optimal Path Control of Manipulators with Bounded Joint Velocities and Torques, Proc. IEEE Int. Conf. on Robotics and Automation, Minneapolis, Minnesota, (1996), pp.1572-1577.

(16) Galicki, M., Time-Optimal Controls of Kinematically Redundant Manipulators with Geometric Constraints, IEEE Trans. on Robotics and Automation, Vol.16, No.1 (2000), pp.89-93.

(17) Galicki, M., The Planning of Robotic Optimal Motions in the Presence of Obstacles, Int. J. of Robotics Research, Vol.17, No.3 (1998), pp.248-259.

(18) Chen, Y. and Desrochers, A.A., A Proof of the Structure of the Minimum-Time Control Law of Robotic Manipulators Using Hamiltonian Formulation, IEEE Trans. on Robotics and Automation, Vol.6, No.3 (1990), pp.388-393.

(19) Kieffer, J., Cahill, A.J. and James, M.R., Robust and Accurate Time-Optimal Path-Tracking Control for Robot Manipulators, IEEE Trans. on Robotics and Automation, Vol.13, No.6 (1997), pp.880-890.

(20) Ma, S., Time Optimal Control of Manipulators with Limit Heat Characteristics of Actuator, Int. J. of Advanced Robotics, Vol.16, No.3 (2002), pp.309-324.

(21) Ma, S., Hirose, S. and Yoshinada, H., Efficient Redundancy Control of Redundant Manipulators, J. of the Robotics Society of Japan, (in Japanese), Vol.14, No.5 (1996), pp.703-709. 